\title{
APPLICATION OF OPTICAL COHERENCE TOMOGRAPHY TO MONITORING THE SUBSURFACE MORPHOLOGY OF ARCHAIC JADES
}

\author{
Chih-Wei Lu, I-Jen Hsu, Hsiang-Chen Wang, Meng-Tsan Tsai, and C. C. Yang \\ Graduate Institute of Electro-Optical Engineering, National Taiwan University, \\ Taipei, Taiwan, R.O.C. \\ (phone) 886-2-23657624 (fax) 886-2-2365263 (E-mail) ccy@cc.ee.ntu.edu.tw \\ Mei-Li Yang \\ Department of Antiquities, National Palace Museum, Shih-Lin, Taipei, Taiwan, 111 R.O.C.
}

Abstract-- We demonstrate the use of optical coherence tomography for scanning archaic jades from the Liangzhu and the Qijia cultures in ancient China for understanding the subsurface structures of the whitening process or the secondary alteration.

In China, beginning in the late Neolithic period, people employed nephrite and other beautiful stones to fashion personal omaments or to create ritual objects for worship. Archaeologically excavated archaic jades manifest different degrees of whitening. Such differences are primarily due to the character of mineral composition, variation in the handling of the stone, and variations in the underground environments of the jade objects. In the whitening process, the mineral composition of the whitening jade does not change, but the structure of cemented condition could become loose. The use of optical coherence tomography (OCT) may provide the subsurface morphology of a whitened archaic jade. Meanwhile, the artificially whitening jade probably was treated either by burning or by the acidification. By using the OCT scanning of such a sample, we may observe the morphology of the burned jade, and can distinguish the phenomena between the naturally and artificially whitened jades. Two OCT systems were built with different light sources for jade scanning, one with central wavelength at $800 \mathrm{~nm}$ and the other at $1250 \mathrm{~nm}$. Figure l(a) shows the photograph of sample B-1, which is an archaic jade object collected from Qijia culture. Figure 1(b) illustrates the OCT scanning image next to the indicated line with system 1 . In this figure, the signal features above the red curve, which corresponds to the sample surface, are attributed to the flat surface of this jade object. With a flat surface, strong reflection or backscattering makes the side-lobes of the OCT interference fringe pattern visible. This fact does not affect the image quality of the sub-surface structure. From this OCT image, one can see the grain structures in the un-whitened regions (dark areas), as indicated with the white arrows. It is interesting to note that at the boundary between the whitened and un-whitened regions, light penetration is quite shallow. This result can be attributed to relatively stronger absorption in this region. We have demonstrated the use of the OCT technique for scanning the sub-surface morphology of jade objects. The two-dimension tomography images were obtained essentially from the refractive index variations in the samples. However, it is believed that material absorption also plays an important role. Several samples of archaic jade objects from Liangzhu culture and Qijia culture were scanned for understanding the sub-surface morphology of the whitening process. Typically, after the whitening process, the sub-surface material became structure-less and provided statistically homogeneous random scattering. Contrast to the original rock structures in the un-whitened regions, OCT scanning in the whitened regions was deeper. The difference of sub-surface morphology between whitened and un-whitened regions should provide us useful information for archaeology study.
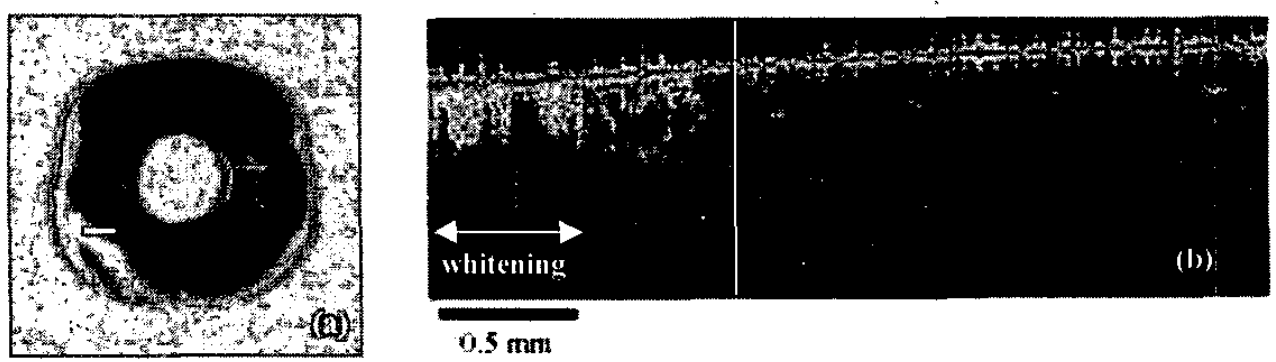

$0.5 \mathrm{~mm}$

Fig. 1 (a) Photograph of sample B-1, which is an archaic jade object collected from the Qijia culture. Part (b) illustrates the OCT scanning image next to the indicated line with system 1. 